\title{
LA CONSTRUCCIÓN DEL SUJETO FEMENINO A TRAVÉS DEL ESPACIO NARRATIVO EN DOS NOVELAS CONTEMPORÁNEAS
}

THE CONSTRUCTION OF THE FEMININE SUBJECT IN NARRATIVE SPACE

\author{
Rosa Ma CALERo JuRAdo \\ Universidad de Córdoba \\ rcalerojurdo@gmail.com
}

\begin{abstract}
RESUMEN: El espacio narrativo es un elemento muy complejo y con gran trascendencia en el análisis narratológico, ya que determina importantes interacciones con otros códigos ideoculturales y extratextuales. El objetivo de este estudio es analizar la configuración del discurso espacial, en tanto que localización y ámbito de actuación, siguiendo los estudios de Valles Calatrava, y cómo este revela una imbricación manifiesta en la construcción del sujeto. Concretamente, la investigación se centra en la construcción del sujeto femenino desde la perspectiva de la differance francesa con el objetivo de comprobar que ciertos escenarios serán determinantes parar romper con el discurso patriarcal hegemónico y contribuir en la formación de nuevo orden simbólico a través de diversos sujetos femeninos.
\end{abstract}

Palabras Clave: Novela, espacio, identidad, sujeto, femenino

ABSTRACT: The narrative space is a complex and significative element within narratological analysis since it may determine key interactions among Ideo-cultural and extratexual codes. The main aim of this research is to analyse how spatial discourse is drafted. According to what Valles Calatrava states, it should be taken into account not only the position or the course of action but also, as he reveals, a manifest imbrication in the subject structure. Specifically, this research focuses in the construction of the feminine subject from the French perspective of différance in order to both verify the importance of certain contexts to break hegemonic patriarchal discourse as well as to contribute to the formation of a new symbolic structure by including several feminine subjects.

KeyWords: Novel, Space, Identity, Subjet, Femenine

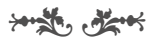




\section{INTRODUCCIÓN}

Las investigaciones sobre género propuestas por el feminismo académico son material indispensable en los Estudios Culturales. Uno de los objetivos principales de dichas investigaciones es mostrar un concepto claro del androcentrismo patriarcal para su consecuente deconstrucción. Esta forma de discriminación ha sido fielmente reflejada por las investigaciones sobre la teoría literaria feminista, cuyo objetivo es estudiar las conexiones entre texto, género e identidad.

De este modo, a través del análisis literario podemos ver cómo las identidades de género se forman mediante un proceso de socialización esencialmente masculinizado y que responde al modelo patriarcal, por lo que la identidad de la mujer queda definida no como sujeto, sino como objeto de uso masculino. La imposibilidad para las mujeres de construir una identidad propia nos muestra la dominación masculina evidente, por la que lo femenino queda construido en función de las necesidades masculinas, pero no como sujeto independiente. ${ }^{1}$

\section{Aspectos teóricos}

Dentro de los enfoques feministas de los Estudios Culturales, el feminismo de la diferencia trata de mostrarnos cómo se construyen los discursos de poder en la construcción de la identidad masculina. Para ello, autoras como Julia Kristeva (2003) o Luce Irigaray (1982) utilizan herramientas del psicoanálisis para acercarse a los textos literarios ${ }^{2}$ y mostrar el proceso de construcción de las identidades patriarcales en los diversos modelos de mujer. Esto permite al análisis de la diferencia abandonar los "sujetos genéricos y universales" hegemónicos del androcentrismo. Gracias al distanciamiento de los sistemas estructurales machistas, las mujeres pueden reconstruir un relato propio y en primera persona de su identidad.

Para poder reconstruir ese sujeto o construir una identidad propia de mujer, estas autoras apuestan por la creación de orden simbólico, y una de las herramientas que utilizan para ello es lo que denominan la "escritura femenina" (o "écriture féminine"), que puede ser adoptada tanto por hombres como por mujeres y es capaz de analizar ese estado cambiante del sujeto hasta lograr su propia construcción e identidad.

En esta investigación, el objetivo es analizar la creación de ese orden simbólico a través del discurso con el que se construye el espacio narrativo, en tanto que la mujer ha sido capaz de reflejar su situación en el mundo gracias a la escri-

\footnotetext{
1 Simone de Beauvoir expone en su afamada obra El segundo sexo (Beauvoir, 1949) cómo la dominación masculina atribuye a los hombres la libertad, el poder de la producción y la trascendencia, mientras que la mujer aparece encadenada a un esencialismo naturalista cuyo fin es sostener esa idea del hombre hacedor. Esta construcción dominante se sistematiza con el rol de género asociado "naturalmente" a las mujeres, que históricamente han sido relegadas a lo íntimo o lo privado.

2 El feminismo de la diferencia parte de una base lacaniana rectificada para dar cabida a un imaginario femenino más independiente, construyéndose así los diferentes "modelos" o sujetos de mujer.
} 
tura literaria. En ella, el lenguaje es una herramienta fundamental para deconstruir el orden impuesto y crear una alternativa simbólica diferente. La narrativa es un modo de expresión idóneo para esta tarea, pues no hay que olvidar que el acto de narrar implica el desarrollo de una posición y una responsabilidad del sujeto, entendiéndolo como la escritura del yo o nosotros. Dentro de esta narración, el espacio ayuda a la comprensión de los mecanismos que determinan el funcionamiento de la realidad circundante y su constitución precisa de un sujeto que lo ocupe. De este modo, la relación resultante del sujeto con el medio será la base de su identidad.

Autores como Zoran (1984), Bachelard (1957) o Ricardo Gullón (1980) han realizado estudios acerca de los espacios simbólicos, metafóricos o han establecido oposiciones binarias que permiten evidenciar las imbricaciones existentes entre estos espacios y su influencia en el plano ideológico o psicológico. Weisgerberg (1978) ya mostró cómo el espacio afecta, sobre todo, a los acontecimientos y a los personajes, configurando la identidad de estos. De esta manera, no solo se trata de un discurso de información situacional, sino que establece una serie de funciones distribucionales o sintácticas que se corresponden con los hechos y con los actos de los personajes. Por tanto, el espacio tiene una relación metonímica con los personajes pues puede proyectar su situación emocional.

Para realizar este análisis, se ha tenido en cuenta el estudio pormenorizado del espacio narrativo que Valles Calatrava (2008) lleva a cabo basándose en las aportaciones de Zoran. Así, el acercamiento al espacio lo realiza a través de tres dimensiones: la primera enmarca los hechos relatados en una localización, ya sea real o imaginaria, atendiendo al nivel situacional, es decir, se trata del emplazamiento de los hechos o los seres; la segunda está relacionada con la dimensión escénica o ámbito de actuación y supone una ordenación narrativa de los acontecimientos, pues distribuye los hechos y a los personajes en escenarios; finalmente, hay que atender a la configuración espacial en el discurso, en otras palabras, cuáles son las operaciones verbales y codificaciones que producen el texto del espacio.

Así, la relación entre espacio e identidad femenina ha sido recalcada tradicionalmente. Entre otros estudiosos, destaca Doreen Masey (1994) quien afirma, en su teoría de las esferas separadas, que lo público (la calle o el exterior) ha sido el lugar ocupado por los hombres, mientras que la mujer ha sido relegada a lo privado (el hogar). Por tanto, el discurso narrativo también ha mantenido a la mujer en ese espacio interior del que pretende salir para construir orden simbólico. En este sentido, la propia Julia Kristeva (2004) entiende el texto narrativo como la práctica que pone en juego la situación del sujeto en la lengua, esto es, la manera de verbalizar la realidad de las mujeres, sometidas todavía a una interpretación privada del espacio y cuya creación discursiva también obedece a ese androcentrismo hegemónico impuesto.

Esta construcción de la identidad femenina a través de los espacios narrativos se ejemplificará, a continuación, con el análisis de dos novelas contemporáneas: La plaza del diamante, de Mercè Rodoreda e Irse de casa, de Carmen Martín Gaite. Estas obras literarias han sido elegidas por sus protagonistas y escritoras 
mujeres, en primer lugar; pero también por la diversidad de modelos de mujer que configuran en el devenir de la de/reconstrucción de sus identidades. Por otro lado, se trata de dos novelas contextualizadas en dos momentos históricos muy diferentes y muy decisivos para la historia de España, ya que Colometa desarrolla su historia en los aledaños de la Guerra Civil, mientras que Amparo lo hace una vez pasada la transición.

\section{La metamorfosis íntima de "Colometa" (La plaza del diamante, de Mercè RODOREDA)}

Siguiendo la idea de los múltiples sujetos de mujer que se construyen desde el análisis feminista de la diferencia, uno de estos ejemplos de identidad puede ser más intimista como sucede en La plaza del diamante (1986), de Mercè Rodoreda. Se trata de una novela en la que los espacios que constituyen el ámbito de actuación juegan un papel fundamental en el proceso de deconstrucciónconstrucción de la protagonista, lo que se analizará a continuación. Sin embargo, también hay que atender a la localización: Barcelona, un espacio real que se descubre ya desde el título, pues alude a una plaza real que existe en el trazado urbano de la ciudad, pero también a otros lugares muy conocidos como el Parque Güell, la catedral de la Sagrada Familia o la casa "La Pedrera". El más importante y significante es la propia "plaza del diamante", el lugar donde conoce a "el Quimet", quien, allí mismo, le arrebata su nombre (Natalia) para imponerle el de "Colometa" ("palomita" en catalán). Esta primera aparición del espacio público evidencia la dominación masculina en la que el constructo mujer supone el objeto del hombre (su "palomita") y, además, la condiciona en la forma de entrar en el apartamento que el Quimet compra para que convivan, casi una imposición oculta. Aquí comienza el proceso de metamorfosis que su identidad va a experimentar, pues no solo transforma su nombre, sino que además ve cómo el apartamento se llena poco a poco de palomas, ocupando también todo el espacio privado que sí es tradicionalmente el que corresponde a la mujer.

Por tanto, su identidad no solo se ha visto eliminada por ese proceso de socialización masculino que hace que la mujer deje su trabajo en el exterior y se quede en casa cuidando de su familia, como ya le sucede a la protagonista, sino que su espacio privado de "privilegio" femenino también se ve ocupado por los animales/objeto que ella representa para su marido. Así se observa en los siguientes fragmentos:

Hicimos el palomar. [...] Me vaciaron la buhardilla de todo lo que yo tenía allí: el cesto de la ropa, las sillas medianas, el canasto de la ropa sucia, el cestillo de las pinzas... -A la Colometa la estamos echando de casa. Me prometieron que, más adelante, me harían un sotechado para poner mis cosas, pero de momento lo tuve que bajar todo al piso, y si quería ir al terrado a sentarme un rato tenía que subir con la silla. Dijeron que, antes de dejar salir a las palomas tenían que pintarlo. [...] Lo pintaron de azul y el pintor fui yo. (Rodoreda 1986: 75-76)

Y más adelante ocupan toda su casa: 
Las palomas, cuando estuvieron cansadas de volar, fueron bajando ahora una y luego otra, y se metieron en el palomar como viejas en misa, con pasos menuditos y con la cabeza adelante y atrás como maquinitas bien engrasadas. Y desde aquel día no pude tender la ropa en la azotea porque las palomas me la manchaban. La tenía que tender en el balcón. Y gracias. (Rodoreda 1986: 83)

De esta manera, observamos cómo el sujeto narrativo tampoco puede construir su discurso íntimo de lo privado, lo que hace que Colometa se sienta más y más vacía a lo largo del desarrollo de los acontecimientos, pues no podrá deconstruir el orden hegemónico patriarcal impuesto, ya que ese le es también arrebatado y este estado la lleva a destruir ese palomar para así poder conquistar su propio espacio privado, como bien se expresa en el discurso:

La buhardilla de la azotea para mí, la trampilla tapada, las sillas dentro de la buhardilla, el camino de las palomas cerrado, el cesto de la ropa en la azotea, la ropa tendida en la azotea. Los ojos redondos y los picos afilados, el tornasol malva y el tornasol manzana, ¡todo a paseo! (Rodoreda 1986: 134)

Tras el estallido de la Guerra Civil y la salida de "el Quimet" al frente, Colometa comienza a trabajar en la casa de unos señores de la clase social alta catalana. Ese trabajo, después de que su marido no vuelva de la guerra y no tenga esa opresión masculina tan cercana, puede considerarse la función liberadora que el feminismo ha visto en la salida de la mujer al mundo laboral. Sin embargo, la protagonista es discriminada por su situación de mujer asolada por las consecuencias de la guerra y así lo expresa en su discurso. Esta casa es totalmente diferente a su apartamento, de ahí su obsesión por describir todos los espacios y muebles, las ventanas y la luz que había y, especialmente, la decoración arquitectónica. Sus palabras nos muestran su desorientación, lo que marca aún más una inseguridad que le hará más difícil acercarse a una identidad propia para dejar de estar sometida por su marido o por sus jefes, en definitiva, por la sociedad masculinizada en la que vive. Así se muestra en el texto:

Cruzando el patio se pasaba por una galería con techo y el techo de esta galería era el suelo de la galería descubierta del piso que hacía el entresuelo por la parte de arriba. A la galería de abajo daban dos balcones: por uno se entraba al comedor, por el otro se entraba a la cocina. No sé si me explico bien. Y con el señor del guardapolvo, que era el yerno de la casa y era el dueño, entramos en el comedor. Me hizo sentar en una silla contra la pared y encima de la cabeza tenía una ventana que llegaba hasta el techo del comedor que daba media vuelta, pero aquella ventana daba a ras de la calle donde estaba la puertecita del jardín por donde había entrado. (Rodoreda 1986: 95-96)

A partir de entonces, Colometa comienza a recorrer las calles de Barcelona en un recorrido $\sin$ fin que hace que no perciba los lugares públicos que transita. De la misma manera que sucede con la casa de los señores, las calles le producen un estado de nervios del que no es capaz de escapar, por lo que en el discurso nos 
presenta una descripción de espacios laberínticos y que producen en el lector la misma sensación de asfixia que en la propia protagonista:

Otra vez tuvo un tranvía que parar en seco cuando crucé la calle Mayor; el conductor me regañó y vi gente que se reía. Me paré en la tienda de los hules haciendo que miraba, porque a decir verdad debo decir que no veía nada: solo manchas de colores, sombras de muñeca... (Rodoreda 1986: 179)

Esta angustiosa percepción de los espacios públicos, unida a su situación económica, la llevarán a conocer a Antoni, quien le ofrecerá trabajo y con quien acabará casándose. La casa de Antoni también es descrita desde la percepción de nuestra protagonista. Sin embargo, ya no utiliza la descripción enmarañada de la que se valía para la casa de los señores, sino que habla de sencillez y oscuridad, como resulta su vida desde que trabaja allí:

La casa era sencilla y oscura, aparte de dos habitaciones que daban a la calle que iba al mercado. Era así: desde la cortina japonesa hasta el fondo, que era una sala con sofá y butacas con fundas y una consola, había un pasillo. A la izquierda de ese pasillo, dos puertas, una junto a otra, para entrar a las dos habitaciones con ventana que daba a la calle que iba al mercado. Al final del pasillo, la cocina y una habitación sin ventanas, trastienda, almacén, llena de sacos de grano y de sacos de patatas y de botellas. Y en el pasillo nada más. Al final del pasillo, la sala; y a la derecha de la sala, el dormitorio del tendero, tan grande como la sala, con balcón que daba a una galería cubierta por arriba por la galería del primer piso y sostenida por cuatro columnas de hierro. Pasada la galería, un patio polvoriento, separado del jardín del primer piso por una verja con lanzas. (Rodoreda 1986: 198)

Esta nueva casa sí constituirá un espacio privado que le permita construir su espacio de intimidad, empezando por la recuperación de su nombre, Natalia, pero también el espacio de esta casa jugará un papel importante. A diferencia de lo que ocurrió en el apartamento donde vivía con el Quimet, ahora es ella la que decide la decoración de la casa y compra nuevos muebles para ello:

Pero antes de casarnos él hizo arreglar aquella casa. Dije que quería camas de metal para los niños y tuve camas de metal, como la que yo había tenido de soltera y había tenido que vender. Dije que quería cocina colgada y tuve cocina colgada. Dije que quería tapete sin mancha de tinta y tuve tapete sin mancha de tinta. Y un día le dije que yo, aunque fuese pobre, era delicada de sentimientos y que preferiría no llevar a la casa nueva ni una sola cosa de la casa vieja: ni la ropa. Y todo lo tuvimos nuevo y cuando le dije que aunque era pobre era delicada de sentimientos, contestó que él era como yo. Y dijo la verdad. (Rodoreda 1986: 213)

Como se observa en este fragmento, también ha cambiado la forma de articular la voz de Colometa, que ahora habla por sí misma, con lo que se afianza su proceso de deconstrucción-construcción de identidad: 
Poco a poco, y costándome mucho, me iba haciendo la casa mía, las cosas mías. La oscuridad y la luz. Sabía las claridades del día y sabía dónde caían las manchas de sol que entraban por el balcón del dormitorio y de la sala, y cuándo eran largas, y cuándo eran cortas. (Rodoreda 1986: 219)

Sin embargo, Natalia ha conseguido hacer del espacio actuacional que supone la casa de Antoni su casa; en ese espacio privado consigue, al menos, construir su identidad de mujer con la suficiente libertad para poder deconstruirla y reconquistar el espacio público que antes rechazaba. Ese hogar le permite una cierta autonomía real, lo que implica una transición del espacio privado opresor, al espacio privado liberador.

\section{La conquista de la gran ciudad de Amparo Miranda (IRSE DE Casa, De Car- men MARTín Gaite)}

Otro ejemplo de modelo femenino creado a partir de la interpretación del espacio narrativo lo encontramos en la novela Irse de casa (1998), de Carmen Martín Gaite. En este caso, el espacio se une al tiempo en lo que Bajtin (1989: 237-409) llamó "cronotopo". 3 Así, en esta novela de Martín Gaite, Amparo Miranda, la protagonista, ha conseguido romper con la tradición patriarcal que imperaba en la sociedad de su país de origen y se ha construido una vida nueva en Nueva York en la que ella es la dueña de sí misma - de sus decisiones, aciertos, posturas, pensamientos, etc.- y en la que queda muy lejos del espacio privado de la familia patriarcal, ya que ahora trabaja y se mueve en la gran ciudad de manera independiente. Sin embargo, no consigue alcanzar su identidad plena porque sigue presa de un proceso de socialización masculino "innato" en la mujer. Así, aunque ha conseguido acabar con ese orden simbólico hegemónico que relega a la mujer al espacio de la intimidad y de lo privado, no consigue (re)construirse, es decir, crear un orden simbólico sin estar coartada por la propia sociedad y la cultura. Por ello, decide realizar un viaje a su tierra natal, a los espacios que ocupó en él, para analizar y comprender no solo su vida sino las decisiones que la han hecho llegar hasta donde está: ser una diseñadora de gran fama en Nueva York.

En primer lugar, la localización de esta novela implica el pasado y el presente: su pasado está representado simbólicamente en su ciudad natal, mientras que el presente está en la ciudad de Nueva York. En cuanto a la primera, no se conoce el topónimo de la misma, pero la protagonista va describiendo cada uno de los lugares recorre. Esta ciudad de su pasado se muestra diferente, ya que ha sufrido cambios a lo largo de los años y el paisaje que la protagonista observa desde la ventana nada tiene que ver con su recuerdo, algo que le abruma por

\footnotetext{
3 En Teoría y estética de la novela, Bajtin define el "cronotopo" como la conexión esencial de las relaciones temporales y espaciales asimiladas artísticamente en la literatura. En otras palabras, lo plantea como una unidad indisoluble entre espacio y tiempo, ya que se conciben en vinculación con el movimiento y la materia, por lo que se configuran como sus propiedades de modo que el tiempo puede ser una coordenada espacial: la cuarta dimensión del espacio.
} 
miedo a que el tiempo haya borrado ese pasado que viene buscando en este viaje que supone una dialéctica entre al antes y el ahora.

Por otro lado, la segunda localización, que supone el presente de la protagonista, es la ciudad de Nueva York. Se trata del espacio público que la protagonista ha logrado conquistar gracias a su trabajo como modista y creadora de una firma de moda importante en EEUU. Tanto Amparo Miranda como su madre se revelan como mujeres transgresoras en su época, que consiguen deconstruir su identidad de mujeres objeto impuesta que las confinaba al hogar y a los cuidados para "salir de casa" como símbolo de su ciudad natal. Su camino de (re) construcción de una identidad propia se relaciona, entonces, con Nueva York donde conquista el espacio público dedicándose al diseño.

También cobrarán mucha importancia los espacios de la historia (ámbito actuacional) que se vivan en el presente de la narración y que, al mismo tiempo, sean rememorados desde el pasado. En este sentido, el más importante de los integrantes de este ámbito de actuación en la novela de Martín Gaite es la "Plaza del Rincón", el primer lugar visitado por Amparo y sobre el que pivotarán los demás espacios recorridos durante este viaje. Se trata de una zona muy visitada por ella en su juventud porque allí se sentía aislada del exterior, pero además será clave también en el presente, porque desde allí podrá observar ese nuevo exterior y compararlo con el pasado sin ser vista. Así lo desarrolla en el discurso narrativo:

En una triangular que tenía dos banquitos enfrentados en el pico del fondo bajo un árbol como pintado en telón de teatro, solía antaño hacer ella un alto para repasar sus lecciones antes de entrar en la academia de idiomas, pero luego empezó a llegar más pronto y a quedarse más tiempo. Se traía una novela o se entregaba al vicio dilecto, ardiente y oculto, imaginar cómo sería de mayor; [...] Se llamaba la plaza del Rincón. Y cuando ahora se volvió a topar con ella y la reconoció de inmediato, contuvo la respiración unos instantes y miró recelosa alrededor, como temiendo que alguien pudiera ser testigo de tan fundamental reencuentro. Pero no pasaba nadie. Llevaba dos mañanas viniendo tempranito a sentarse allí y seguía sin pasar casi nadie, o los que pasaban no la veían, en eso consistía la magia del lugar, la misma que la llevó de adolescente a elegirlo como escondite, tan inconfundible para ella como invisible para los demás; y recuperar esa sensación de privilegio vino a suponer el primer acontecimiento digno de reseña en su travesía del desierto: "He estado en la plazuela del Rincón y existe", anotó escuetamente por la noche en su agenda. (Martín Gaite 1998: 141-142)

Se trata de un espacio catalizador de ese proceso de deconstrucción ya completado, que le permite la dialéctica pasado-presente necesaria para la (re)construcción de su propia identidad. Desde esta plaza, Amparo se prepara para el choque con la realidad que va a experimentar con este viaje, fruto de una idea de guión cinematográfico para una película que su hijo plantea:

Hay tres puntos clave en ese borrador -se dijo-, uno el encuentro con mi armario de luna, otro la entrada en una casa donde se han demolido todos los 
tabiques que oprimieron mi infancia, y el tercero la voz desgarrada de Olimpia invocando al vacío una respuesta sin esperanza, jrespondedme!, mientras yo me escondía tras un árbol. (Martín Gaite 1998: 287)

Estos tres espacios también forman parte del ámbito actuacional: el primero es el reencuentro con su antiguo armario. Hay que tener en cuenta que, tras mudarse a vivir a EEUU, Amparo y su madre venden o regalan los muebles de su casa. Este es el caso del armario de luna que Amparo tenía en su dormitorio y que regalaron a una trabajadora del taller de su madre. En el presente de la obra, Amparo encuentra su viejo armario en la casa de la hija de esta señora y de la que no sabía nada desde la muerte de su madre:

Lo había reconocido, era su viejo armario de luna, ante el cual había reído y llorado tantas veces, y hablado con voz secreta, porque Olimpia le había metido en la cabeza que era muy sano para la salud hablar con una misma, que equivalía a un ensayo general sobre la vida, aunque luego la vida se vaya por caminos imprevistos. [...]

Intentó irse pero no podía, y cuando quiso darse cuenta ya estaba colocada delante del armario. Una figura borrosa de mujer apareció al otro lado con la cama por telón de fondo. No era capaz de mirarla de frente. Pero escuchó su saludo.

-Hola, te he estado esperando durante mucho tiempo, sabía que tenías que volver. ¿Qué ha sido de tu vida?

A Amparo le temblaba la voz al contestar, unas manos invisibles le oprimían el cuello.

-No se puede hacer el resumen de una vida tan larga en pocos minutos, perdóname, me tengo que ir.

[...]

-Ya entiendo, ahora tendrás otros espejos.

$[\ldots]$

-Sí, tengo otros espejos que me dicen mentiras. Pero a todo hay que acostumbrarse. Me alegro de haber venido. Adiós. (Martín Gaite 1998: 182-184)

En un primer momento, Amparo reconoce su antiguo armario en un espacio diferente al que lo recordaba, pues se trata de otra casa. Pero, además, este la hace rememorar recuerdos del pasado que le impiden mirarse en el espejo que contiene y en el que solía mirarse para hablar consigo misma y representarse (hacerse real). Finalmente, lo consigue y se produce un desdoblamiento del personaje que habla con su propio reflejo, como hacía antaño, convirtiéndose en sujeto y objeto, por tanto es una pieza clave para la (re)construcción de su identidad como sujeto mujer porque deja de ser el objeto del hombre, significándose en este reflejo.

Lo mismo sucede con su antigua casa, el segundo componente del ámbito actuacional en esta novela. Es el lugar más importante para el recuerdo de alguien que se ha visto obligado a abandonarlo, aunque sea para encontrar mejoras en su vida. Así, nuestra protagonista entra en la tienda que han construido en lo que antes era su casa y que se llama "Defectos Especiales", un nombre muy adecuado para calificar este anticuario, un lugar donde se vendían artículos del 
pasado (algunos incluso rotos), pero con un valor muy especial por tratarse de objetos con recuerdo y para el recuerdo:

Era un espacio muy amplio, con dos columnas verdes y paredes color ocre. Resultaba difícil calcular, pero más o menos detrás del respaldo de aquella butaca estuvo el tabique que lindaba con las minúsculas habitaciones de dentro, divididas a su vez por tabiques, jcuánto tabique!; aquí en la parte de delante también hubo uno que separaba el probador del taller de las oficialas. [...] De niña soñaba con demoler todos los tabiques y convertirse en habitante de un lugar grande y silencioso para ella sola, olía a sándalo y se imaginaba allí, rodeada de telas de colores, cosiendo trajes para muñecas con ojos de cristal que luego se escapaban porque tenían mucho que hacer. [...] Ahora, entre varios bibelots alineados sobre una cómoda izquierda, reparó en una muñeca vestida de rosa, con gorrito, que no se podía sujetar de pie porque en lugar de piernas tenía un palo. Se había metido en un bosque nebuloso de presencias ficticias, pero no tenía miedo de perderse, prefería perderse. [...] Volvió la cabeza hacia el fondo. Allí, separado del resto por un biombo de pavos reales, había un rincón a modo de taller con tablero de dibujante y botes de pintura, olía a engrudo; es el sitio donde estuvo un día su dormitorio con el armario de luna. (Martín Gaite 1998: 210-211)

Este espacio también será clave para el despertar de Amparo hacia ese pasado que no es doloroso, pues ella puede recordar y hacerlo eterno, aunque el tiempo se lo haya llevado. En esta vuelta al pasado, a su niñez, nuestra protagonista recuerda los muros que había en su casa y que ahora han desaparecido; lo que representa la opresión que ella sentía en su infancia por la sociedad patriarcal de la época y que ahora ha superado como mujer independiente y con una identidad en construcción.

Al salir de allí, Amparo se dirige al jardín de los Moret, la familia de su mejor amiga, Olimpia, y de su gran amor, Abel Bores. Este lugar será el tercer espacio de la historia que compone el ámbito de actuación. Se trata de un lugar del pasado que nuestra protagonista no puede traspasar para llegar a la casa que hay detrás, ya que no es capaz de entrar a ver a su amiga después de la carta que confesaba que estaba enamorada de ella. Nuestra protagonista describe minuciosamente un jardín totalmente diferente al que había en el pasado, porque no está cuidado y también acusa el paso del tiempo. Lo mismo sucede con su antigua amiga, por la que también han pasado los años:

La fachada de delante tenía todas las ventanas y balcones cerrados. Ni un ruido. Ni una luz. Miró con aprensión la breve escalera custodiada por los dos perros de piedra. Subir aquellos escalones y llamar a la puerta ni se le pasaba por la cabeza, nada de lo que pudieran decirse ella y Olimpia resultaría natural, sino más bien penoso. Recordó súbitamente la primera y única carta que recibió de ella en Ginebra, cuando estaba tratando de abrirse camino antes del traslado a Nueva York. [...] Era una carta muy larga y lacerante. Una carta de amor. [...] No se puede calcular el tiempo cuando echas a volar recuerdos. Avanzó casi con miedo por entre los árboles oscurecidos y se dirigió a la parte trasera del jardín. [...] Por la galería acristalada del segundo piso se paseaba una figura alta 
vestida con traje largo de color oscuro. [...] Llegaba al final de la galería, daba una vuelta y reemprendía su paseo lento, entreverado de paradas y gestos solemnes. Estaba recitando. Amparo agudizó el oído, porque en algunos tramos alzaba más la voz y la mayor parte de las ventanas estaban abiertas. [...] Hasta que la mujer de pelo blanco se paró y se asomó a una de las cristaleras, agitando los brazos. Ahora ya declamaba con voz tan potente que llegaba a los últimos rincones del jardín. Era una voz de timbre varonil: la voz de Olimpia. (Martín Gaite 1998: 216-218)

Después de estos sucesos transcurridos en espacios clave para el recuerdo de nuestra protagonista, decide volver a Nueva York anticipadamente; ya que estos tres acontecimientos le han hecho ver su pasado desde el presente, analizándolo y cerciorándose de que mirar hacia atrás no tiene que ser doloroso para ella, pues ha conseguido subvertir los dogmas patriarcales que la sociedad le imponía y construir una identidad libre de dominación. Tampoco existen ya estos muros en su antigua casa porque ella ha roto y en su lugar hay una tienda nueva, es decir, se ha reconstruido el espacio que ocupaba su casa de la misma manera que ella ha (re)construido su identidad hacia lo público, hacia el exterior. Además, ha visto su propio "yo" del presente reflejado en su antiguo armario y ha sido capaz de reflejarse como objeto y sujeto, por lo que se rompe radicalmente con esa creación cultural impuesta por el androcentrismo.

De esta manera, gracias a la importancia de los espacios de la historia y el cronotopo, que ha jugado con el tiempo pasado y presente para controlar esa dialéctica entre memoria y olvido de los lugares de su infancia, la protagonista de Irse de casa ha finalizado el proceso de deconstrucción-(re)construcción que la mujer necesita para componer su propio relato en primera persona lejos de la imposición masculina de la tradición occidental.

\section{CONCLUSIONES}

Después de llevar a cabo una lectura analítica de dos novelas contemporáneas tan dispares como La plaza del diamante e Irse de casa, el análisis pormenorizado del espacio narrativo muestra una imbricación manifiesta en el proceso de construcción de identidad femenina según lo defienden las teóricas del feminismo de la diferencia. Con respecto a las dos obras citadas, se constata que aparecen dos "modelos" diferentes de mujer: el primero desde lo íntimo y privado del hogar, espacio que la tradición patriarcal le ha reservado a la mujer, y el segundo, desde lo público pero rememorando la intimidad de lo privado a través del tiempo. De esta forma, podemos afirmar que el sujeto mujer necesita deconstruir el modelo hegemónico impuesto conquistando el espacio público, pero de la misma forma, también precisa volver a ese espacio privado para deconstruir su identidad impuesta y comenzar el proceso de construcción de una identidad propia.

Como consecuencia, este proceso de deconstrucción-construcción del sujeto femenino mostrará multitud de modelos de mujer alternativos que escapan del proceso de socialización masculina y que componen un discurso propio que crea orden simbólico libre del poder dominante. 


\section{OBRAS CITADAS}

Bachelard, Gaston (1986): La poética del espacio [1957]. México, FCE.

Bajtin, Mijaíl (1989): Teoría y estética de la novela: trabajos de investigación. Madrid, Taurus. Beauvoir, Simone (1999): El segundo sexo [1949]. Trad. Alicia Martorell. Madrid, Gredos.

Gullón, Ricardo (1980): Espacio y novela. Barcelona, Antoni Bosch.

Irigaray, Luce (2009): Ese sexo que no es uno [1982]. Madrid, Akal.

Kristeva, Julia (2003): El genio femenino: la vida, las locuras, las palabras. Buenos Aires, Paidós.

Martín Gaite, Carmen (2002): Irse de casa [1998]. Barcelona, Anagrama.

Massey, Doreen (1994): Space, Place and Gender. Minneapolis, University of Minnesota Press.

Rodoreda, Mercè (2012): La plaza del Diamante [1986]. Barcelona, Pocket Edhasa.

Valles Calatrava, José R. (1999): El espacio en la novela. El papel del espacio narrativo en "La ciudad de los prodigios" de Eduardo Mendoza. Almería, Grupo de Investigación de Teoría de la Literatura y Literatura Comparada de la Universidad de Almería.

- (2008): Teoría de la narrativa. Una perspectiva sistemática. Madrid, Iberoamericana.

Weisgerberg, Juillet (1978): L'espace romanesque. Lausana, L'âge d’homme.

Zoran, Gabriel (1984): "Towards a Theory of Space in Narrative", Poetics Today, n. ${ }^{\circ}$ 5, pp. 309-335. 